\title{
Stochastic space-time disaggregation of rainfall into DSD fields
}

\author{
by Marc Schleiss and Alexis Berne \\ Laboratoire de Télédétection Environnementale (LTE) \\ École Polytechnique Fédérale de Lausanne (EPFL), Switzerland
}

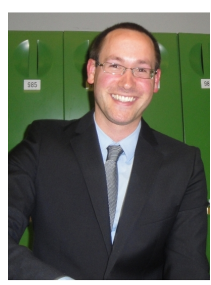

\begin{abstract}
A stochastic method to disaggregate rain rate fields into DSD fields is proposed. It is based on a previously presented DSD simulator that has been modified to take into account prescribed rain rate values at a coarser scale. The quantity used to drive the disaggregation process can be the rain rate, the radar reflectivity or any variable directly related to the DSD. The method is illustrated using radar rain-rate data provided by MeteoSwiss. The possibility to drive the disaggregation process using multiple constraints (e.g. the reflectivity at different frequencies or polarizations) is discussed at the end of the paper.
\end{abstract}

\section{Introduction}

The investigation of many natural hazards and processes like flash floods and landslides requires accurate rain rate estimates at high spatial and temporal resolutions (in the order of $1 \mathrm{~km}$ and $5 \mathrm{~min}$ or higher). So far, only weather radars are capable of providing such rainfall estimates. Sometimes, the resolution of radar rain-rate estimates is, however, insufficient. The available measurements must then be downscaled to higher resolutions. This process is called disaggregation. Unlike aggregation, disaggregation is not uniquely defined and generally admits different solutions. Disaggregated rainfall fields should therefore be seen as particular realizations of a stochastic process satisfying a set of conditions (e.g., the preservation of the space-time structures and the mean areal rain rates).

Many different disaggregation methods of various degrees of complexity (both deterministic and stochastic) have been proposed in the literature. Among the few deterministic disaggregation methods, one can mention the linear model by Ormsbee (1986), the chaotic dynamical approach by Sivakumar et al. (2001) and the QQ transform by Bárdossy and Pegram (2011). So far, most of the stochastic disaggregation methods focus on the disaggregation of rain rate time series using stochastic point processes (e.g., Hershenhorn and Woolhiser, 1987; Koutsoyiannis and Onof, 2001) or (multi)fractal random cascades (Olssen and Berndtsson, 1998; Guntner et al., 2001). Different approaches based on simulated annealing (Bárdossy, 1998) and master target scaling techniques (Jennings et al., 2010) are also worth mentioning. A lot of efforts have also been devoted to spatial and spatiotemporal disaggregation methods, like the morphological algorithm by Kumar and Foufoula-Georgiou (1994), the Haar wavelet transform proposed by Perica and Foufoula-Georgiou (1996), the Gibbs sampler by Onibon and Lebel (2004) and filtered autoregressive models by Rebora et al. (2006).

All the methods mentioned above are primarily concerned with the disaggregation of rain rate fields. In many remote sensing applications, alternative and more general parameterizations of rainfall such as the (rain)drop size distribution (DSD hereinafter) can be more valuable than the rain rate. The objective of this article is to extend previous work on rainfall disaggregation by proposing a new disaggregation method that includes the DSD as an outcome.

\section{Models}

\subsection{DSD model}

The DSD is assumed to be adequately described by a combination of a concentration parameter $N_{t}\left[\mathrm{~m}^{-3}\right]$ and a probability density function $f$ :

$$
N(D)=N_{t} f(D)
$$

where $N(D) d D\left[\mathrm{~m}^{-3}\right]$ denotes the number of drops per unit volume with equivolume spherical diameters between $D[\mathrm{~mm}]$ and $D+d D$. According to the literature, $f$ can be approximated by a two-parameter Gamma distribution (Ulbrich, 1983; Willis, 1984):

$$
N(D)=N_{t} \alpha D^{\mu} e^{-\Lambda D},
$$


where $\alpha=\left(\int_{D_{\min }}^{D_{\max }} D^{\mu} \mathrm{e}^{-\Lambda D} d D\right)^{-1}\left[\mathrm{~mm}^{-(\mu+1)}\right]$ is a normalization factor taking into account the finite range of possible drop sizes between $D_{\min }[\mathrm{mm}]$ and $D_{\max }[\mathrm{mm}]$. The Gamma DSD model is characterized by three parameters: the shape $\mu>-1[-]$, the rate $\Lambda>0\left[\mathrm{~mm}^{-1}\right]$ and the concentration $N_{t}$. Note that only $\mu$ and $\Lambda$ are used to describe the size distribution of the drops. Because of the natural variability of the DSD, $\left(\mu, \Lambda, N_{t}\right)$ is usually considered as a tri-variate random function in space and time. Simulating DSD fields therefore means generating random (correlated) realizations of $\left(\mu_{x, t}, \Lambda_{x, t}, N_{t_{x, t}}\right)$ at each point $x$ in space and $t$ in time.

Simulating DSD fields is more interesting and general than simulating rain rate fields. In fact, the rain rate can be derived from the DSD, through direct numerical integration:

$$
R=\frac{6 \pi}{10^{4}} \int_{D} D^{3} v(D) N(D) d D
$$

where $R\left[\mathrm{~mm} \mathrm{~h}^{-1}\right]$ denotes the rain rate and $v(D)\left[\mathrm{ms}^{-1}\right]$ the terminal fall speed of a drop of diameter $D$. Similar expressions exist for many other quantities like the median drop diameter, the radar reflectivity, the differential reflectivity and the specific attenuation on propagation. Consequently, any quantity that can be derived from the DSD can be used as a driving variable in the disaggregation process. For illustration purposes however, the main variable used in this paper will be the rain rate.

\section{$2.2 \quad$ Intermittency}

Rainfall intermittency, i.e., the presence or absence of rainfall, is described by an indicator function:

$$
I(x, t)= \begin{cases}1 & \text { if } R(x, t)>0 \\ 0 & \text { else }\end{cases}
$$

where $R(x, t)\left[\mathrm{mm} \mathrm{h}^{-1}\right]$ represents the rain rate at location $x \in \mathbb{R}^{2}$ and time $t \in \mathbb{R}$. By extension, we define

$$
I(X, T)=\left\{\begin{array}{l}
1 \text { if } \exists(x, t) \in(X, T) \text { with } I(x, t)=1 \\
0 \text { else }
\end{array}\right.
$$

for a given domain $X$ and period $T$. For simplicity, only square areas of size $k \times k\left[\mathrm{~km}^{2}\right]$ and continuous periods $[t, t+\tau]$ of duration $\tau[\mathrm{h}]$ are considered. In the following, we will use $(k, \tau)$ to denote the space-time scale corresponding to blocks of size $k \times k \times \tau$.

A very important problem that needs to be addressed during the disaggregation process is the fact that rainfall intermittency changes depending on the scale at which it is considered. Statistical analysis of rain gauge and radar data have shown that the rainfall probability increases according to a scaled exponential law of the spatial and temporal scale (Schleiss et al., 2011). Unfortunately, estimating the percentage of dry pixels at the fine scale as a function of the observed percentage of dry regions at the coarse scale is not straightforward. In the following, the authors propose a simple method based on climatological relationships. Assume that we are disaggregating a rainfall field from a coarse scale $\left(k_{1}, \tau_{1}\right)$ to a fine scale $\left(k_{0}, \tau_{0}\right)$ with $k_{0} \leq k_{1}$ and $\tau_{0} \leq \tau_{1}$. According to Schleiss et al. (2011), the dry probability due to the change of scale increases by:

$$
\Delta p=p\left(k_{0}, \tau_{0}\right)-p\left(k_{1}, \tau_{1}\right)>0
$$

where

$$
p(k, \tau)=\left(p_{0}+p_{1} k\right) \exp \left\{-\left[\frac{\tau}{a k^{b}}\right]^{\beta}\right\}
$$

represents the climatological probability that a domain of size $k \times k \mathrm{~km}^{2}$ remains dry for at least $\tau$ hours and $p_{0}, p_{1}, a, b$ and $\beta$ are fixed parameter values provided in Table 1 of Schleiss et al. (2011). The percentage $p[-]$ of dry regions at the fine scale is then estimated as follows:

$$
\hat{p}=\min \{P+100 \Delta p, 1\}
$$

where $P[-]$ denotes the percentage of dry blocks at the coarse scale $\left(k_{1}, \tau_{1}\right)$. A simple numerical application of this procedure shows that the percentage of dry regions increases by about 5 percent points when disaggregating from $5 \times 5 \mathrm{~km}^{2}$ and $15 \mathrm{~min}$ to $1 \times 1 \mathrm{~km}^{2}$ and $5 \mathrm{~min}$. In other words, if the observed percentage of dry blocks at the coarse scale is $5 \%$, it will be about $10 \%$ at the fine scale. However, because $\Delta p$ is estimated using climatological relationships, there might be significant differences between the "real" value of $p$ and its estimate $\hat{p}$. The authors therefore suggest to generate not a single but many different realizations with different values of $\hat{p}$ according to a given tolerance level $\varepsilon_{p}^{\text {abs }}$ (e.g., $5 \%$ around the estimated climatological value). 


\section{$2.3 \quad$ Space-time structure}

The space-time structures of the parameters to be simulated are modeled using variograms, a key tool in the field of Geostatistics. Each DSD parameter has its own variogram. The general form of the space-time structure is, however, identical for all variables:

$$
\gamma_{Z}(h, \tau)=\gamma_{Z}^{(S)}(h-\tau v)+\gamma_{Z}^{(T)}(\tau)
$$

where $h \in \mathbb{R}^{2}$ is a spatial separation vector, $\tau \in \mathbb{R}$ a given time lag and where $\gamma_{Z}^{(S)}$ and $\gamma_{Z}^{(T)}$ are two variograms representing the spatial and the temporal structure of $Z$. In other words, the space-time structure can be decomposed into spatial and temporal variations, linked by a speed and direction of advection $v$.

The fact that, in the absence of any external source of information, the space-time variograms of the DSD parameters must be "guessed" or estimated from the observed structures at the coarse scale is an inherent property of any disaggregation method and should not be seen as a major limitation. Climatological variograms are useful in this context but are not always optimal. In case the spatial and temporal structures at the fine scale are highly uncertain, it is always possible to generate different realizations of the same event using different space-time variogram models covering a wide range of plausible structures.

\subsection{DSD simulation algorithm}

The procedure used to simulate the DSD fields at the fine scale is described in detail in Schleiss et al. (2012) and will not be repeated here. It consists of two main parts: (1) the simulation of an indicator field with prescribed mean and space-time structure to determine the dry/rainy regions in the simulation domain and (2) the simulation of 3 correlated random fields (also with prescribed mean and space-time structure), one for each DSD parameter $\mu, \Lambda$ and $N_{t}$. The algorithm used to generate the rainfall indicator field is the so-called sequential indicator simulation algorithm (SIS). The one used to simulate the DSD parameters is the sequential Gaussian simulation algorithm (SGS). Both algorithms are very popular and extensively documented in the literature.

\section{Disaggregation}

\subsection{Conditions and constraints}

In the following, the conditions that need to be satisfied by the disaggregated fields are described. For simplicity, the driving variable is supposed to be the rain rate. Suppose a target rain rate field $R=\left(R_{1}, \ldots, R_{n}\right)$ consisting of $n$ space-time blocks of size $k_{1} \times k_{1}\left[\mathrm{~km}^{2}\right]$ and duration $\tau_{1}[\mathrm{~h}]$ is provided at the coarse scale. This scale will be denoted by $\left(k_{1}, \tau_{1}\right)$ in the following. In the classical disaggregation framework, the objective is to generate a new rain rate field $r=\left(r_{1}^{(1: m)}, \ldots, r_{n}^{(1: m)}\right)$, at a smaller scale $\left(k_{0}, \tau_{0}\right)$, such that

$$
\frac{1}{m} \sum_{j=1}^{m} r_{k}^{(j)} \in\left[R_{k}-\varepsilon_{R}^{\mathrm{abs}}, R_{k}+\varepsilon_{R}^{\mathrm{abs}}\right] \quad \forall k=1, \ldots, n
$$

where $m$ represents the number of space-time pixels of size $k_{0} \times k_{0} \times \tau_{0}$ per block of size $k_{1} \times k_{1} \times \tau_{1}$ and $\varepsilon_{R}^{\text {abs }}\left[\mathrm{mm} \mathrm{h}^{-1}\right]$ denotes the uncertainty associated to the rain rate measurements at the coarse scale. The disaggregation method proposed in this paper is more general than the classical approach described above. First, a DSD field $N(D)=\left\{N(D)_{1}^{(1: m)} \ldots, N(D)_{n}^{(1: m)}\right\}$ is generated at the fine scale. The corresponding rain rates are then derived from the simulated DSD using Equation (3).

Finally, recall that the generated DSD fields are intermittent. If $I=\left(I_{1}, \ldots, I_{n}\right)$ denotes the rainfall indicator field $\left(0=\right.$ dry $; 1=$ rainy) at the coarse scale and $i=\left(i_{1}^{(1: m)}, \ldots, i_{n}^{(1: m)}\right)$ the rainfall indicator field at the fine scale, the following conditions related to the presence and absence of rainfall need to be satisfied:

$$
\begin{cases}\sum_{j=1}^{m} i_{k}^{(j)}=0 & \forall \text { blocks for which } I_{k}=0 \\ \sum_{j=1}^{m} i_{k}^{(j)}>0 & \forall \text { blocks for which } I_{k}=1 .\end{cases}
$$

The percentage $p_{\text {sim }}$ of simulated dry pixels at the fine scale must also be related to the observed percentage of dry blocks at the coarse scale, according to Equations (7) and (8):

$$
p_{\text {sim }}=1-\frac{100}{n m} \sum_{k=1}^{n} \sum_{j=1}^{m} i_{k}^{(j)} \in\left[\hat{p}-\varepsilon_{p}^{\mathrm{abs}}, \hat{p}+\varepsilon_{p}^{\mathrm{abs}}\right]
$$


This defines the constraints (Equations 10-12) that must be satisfied by the generated DSD fields at the fine scale in order to be consistent with the coarse scale.

\subsection{Disaggregation algorithm}

In the following, the iterative procedure used to generate intermittent DSD fields satisfying the disaggregation constraints given in Equations (10)-(12) is described. For a visual illustration of this algorithm, see Figure 1.

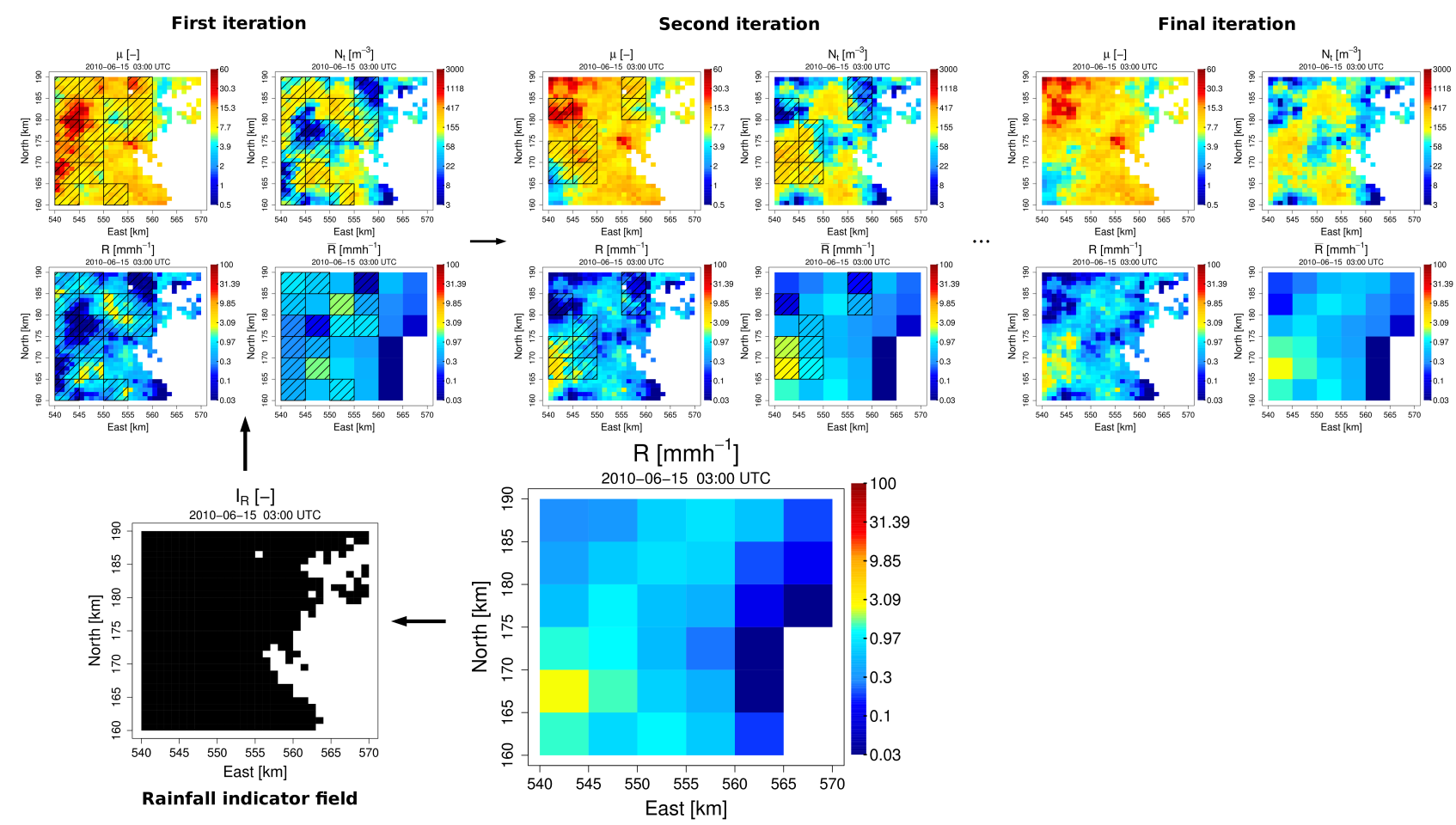

Figure 1: Simplified visual illustration of the disaggregation procedure (without temporal dimension and $\Lambda$ parameter). Blocks with incorrect values of rain rate are shaded in black. Their number decreases with the number of iterations. The procedure continues until all blocks satisfy the conditions given in Equations (10), (11) and (12).

1. Use SIS to generate a rainfall indicator field $i=\left(i_{1}^{(1: m)}, \ldots, i_{n}^{(1: m)}\right)$ with prescribed mean and space-time variogram. Use the dry blocks at the coarse scale to condition the simulations at the fine scale.

2. Use the method described in Schleiss et al. (2012) to generate a random DSD field $\left(\mu, \Lambda, N_{t}\right)$ with prescribed space-time structure.

3. Compute the rain rates $r_{k}^{(j)}$ from the simulated DSD fields using Equation (3). Derive the aggregated rain rates at the coarse scale by averaging the values of $r_{k}^{(j)}$ over each block $k$.

4. Identify all blocks satisfying Equation (10). If all aggregated rain rates are correct, stop.

5. Generate new realizations of $\left(\mu, \Lambda, N_{t}\right)$ on the sub-domain defined by all the (wet) pixels with incorrect block-averaged rain rates. Use the previously simulated values of $\left(\mu, \Lambda, N_{t}\right)$ with correct block-averaged rain rates as conditioning values for the new simulations.

6. Continue until all blocks satisfy Equation (10).

Because the proposed procedure is stochastic, an infinite number of realizations satisfying the same conditions can be generated by repeating the previous simulation steps.

\section{Application}

The purpose of this section is to illustrate the proposed disaggregation procedure for a stratiform and a convective event. In both cases, the variable used to drive the disaggregation process is the rain rate, taken from the operational MeteoSwiss radar rain-rate product over a chosen domain of size $30 \times 30 \mathrm{~km}^{2}$. The original resolution of the radar data is $1 \times 1 \mathrm{~km}^{2}$ and $5 \mathrm{~min}$. For validation purposes, the rain rate values are first 
aggregated over blocks of $5 \times 5 \mathrm{~km}^{2}$ and $15 \mathrm{~min}$ before they are used to drive the disaggregation process (back to the original resolution of $1 \times 1 \mathrm{~km}^{2}$ and $5 \mathrm{~min}$ ). In this way, it is possible to rigorously compare the statistics of the measured and disaggregated fields (not shown to keep the paper short). In this application, the spacetime structures of the DSD parameters are modeled using climatological variograms (for each type of rainfall) derived from a large dataset of DSD measurements at EPFL, Switzerland (Jaffrain et al., 2011). Examples of a disaggregated rain rate field (with corresponding DSD parameters $\mu$ and $N_{t}$ ) are given in Figure 2 (stratiform event) and Figure 3.
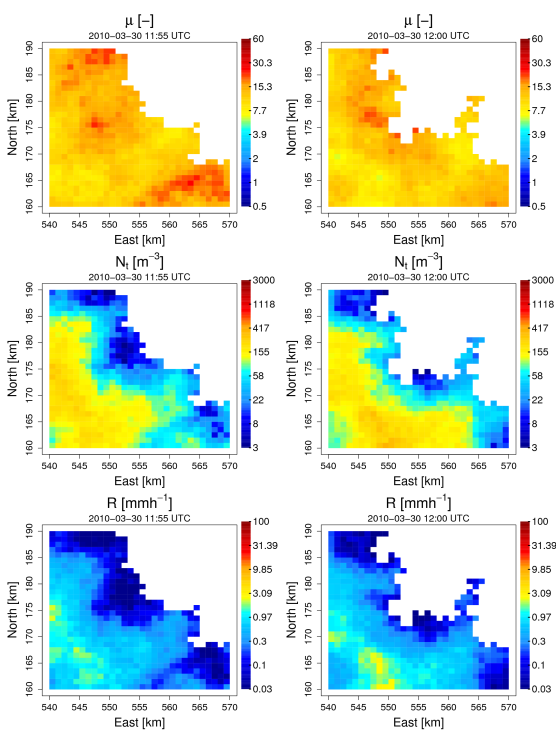

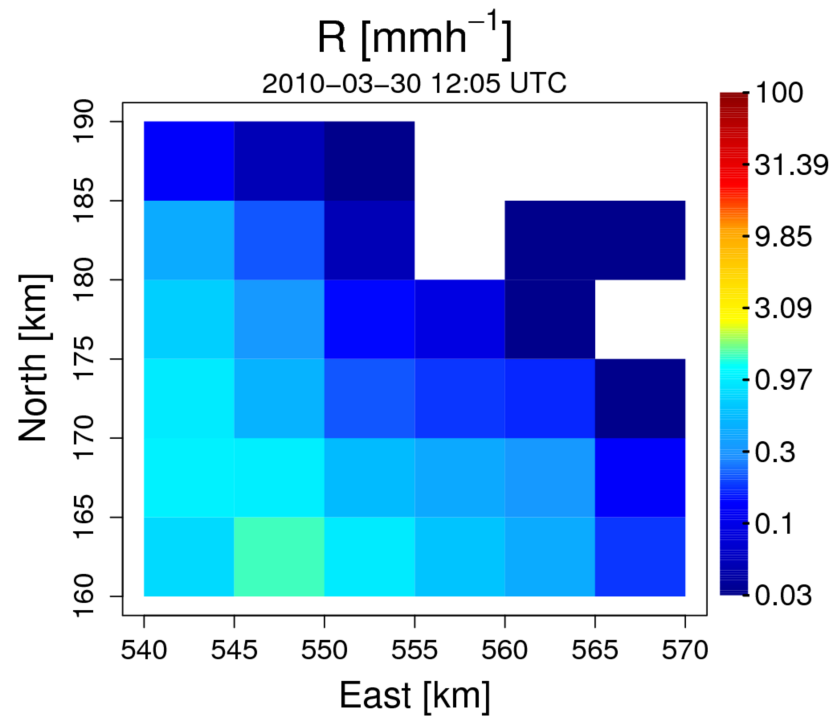

Figure 2: Disaggregated fields of $\mu$ (top row), $N_{t}$ (medium row) and corresponding rain rates $R$ (bottom row) for the first 15 min of event 1 (stratiform). The rate parameter $\Lambda$ is not shown because highly correlated to $\mu$. Each column represents a period of 5-min between 11.55 and 12:05 UTC. The coarse-scale rain rate field at $5 \times 5 \mathrm{~km}^{2}$ and 15 min used to drive the disaggregation process is shown on the right. By construction, the 3 disaggregated rain rate fields at the bottom aggregate exactly into the coarse scale rain rate field.
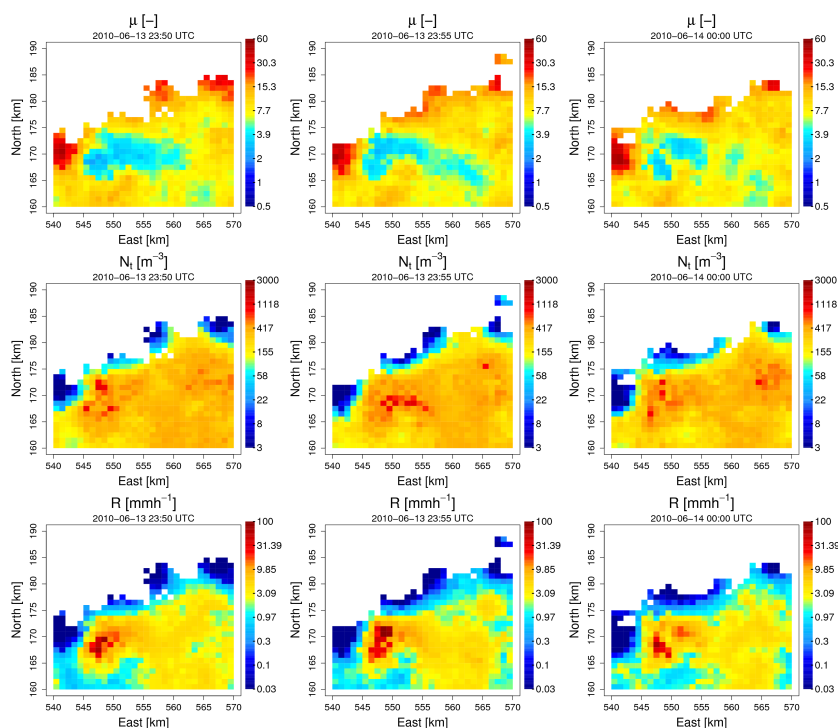

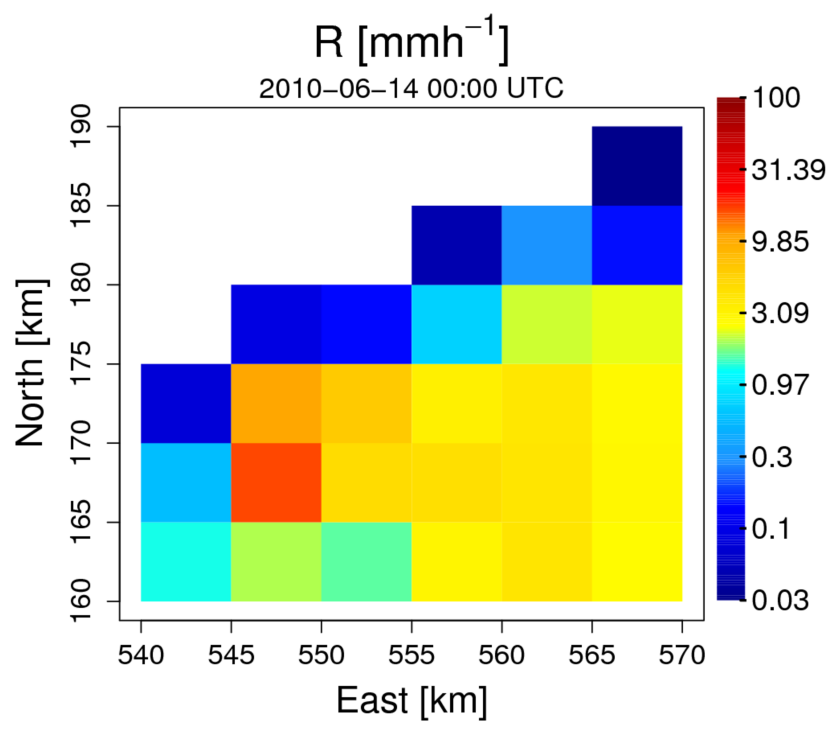

Figure 3: Disaggregated fields of $\mu, N_{t}$ and $R$ for the first 15 min of event 2 (convective). Same format as in Fig. 2.

\section{Conclusions}

In this article, a stochastic method for the disaggregation of rain rate fields into intermittent fields of DSD with given space-time integral rain rates has been presented. The method has been illustrated using radar rain-rate data for two different events (convective vs stratiform). The rain rates derived from the simulated DSD fields 
exhibit a realistic space-time structure and are consistent with radar observations at the same scale. The main advantage of the proposed disaggregation method is the fact that it directly simulates the DSD. It is therefore more general than traditional disaggregation methods which focus on rain rates only. As a consequence, any quantity that is directly related to the DSD (e.g., the rain rate or the radar reflectivity) can be used to drive the disaggregation process. Because it is stochastic, the proposed method can be used to generate large numbers of different realizations for a single input field. Another advantage of the proposed disaggregation method is that the transitions between the individual blocks at the coarse scale are barely visible. This is a clear improvement over many other and previous disaggregation techniques (e.g., random cascades). The main limitation of the proposed disaggregation method is that it is limited to 2-dimensional fields of liquid precipitation. Issues related to mixed-phase precipitation and vertical variabilities (which are very important for satellites applications) are not addressed. Future work will mostly focus on this issue and on the possibility to use dual-frequency reflectivity constraints to drive the disaggregation process.

\section{Acknowledgements}

The authors acknowledge the financial support from the Swiss National Science Foundation (grant 200020132002) and thank Joel Jaffrain for providing the DSD data used to parameterize the climatological variograms.

\section{References}

Bárdossy, A., 1998: Generating precipitation time series using simulated annealing. Water Resour. Res., 34 (7), 1737-1744, doi:10.1029/98WR00981.

Bárdossy, A. and G. Pegram, 2011: Downscaling precipitation using regional climate models and circulation patterns toward hydrology. Water Resour. Res., 47 (W04505), doi:10.1029/2010WR009689.

Guntner, A., J. Olsson, A. Calver, and B. Gannon, 2001: Cascade-based disaggregation of continuous rainfall time series: the influence of climate. Hydrol. Earth Syst. Sci., 5 (2), 145-164.

Hershenhorn, J. and D. A. Woolhiser, 1987: Disaggregation of daily rainfall. J. Hydrol., 95, 299-322.

Jaffrain, J., A. Studzinski, and A. Berne, 2011: A network of disdrometers to quantify the small-scale variability of the raindrop size distribution. Water Resour. Res., 47, W00H06, doi:10.1029/2010WR009872.

Jennings, S. A., M. F. Lambert, and G. Kuczera, 2010: Generating synthetic high resolution rainfall time series at sites with only daily rainfall using a master-target scaling approach. J. Hydrol., 393 (3-4), 163-173, doi:10.1016/j.jhydrol.2010.08.013.

Koutsoyiannis, D. and C. Onof, 2001: Rainfall disaggregation using adjusting procedures on a poisson cluster model. J. Hydrol., 246 (1-4), 109-122, doi:10.1016/S0022-1694(01)00363-8.

Kumar, P. and E. Foufoula-Georgiou, 1994: Characterizing multiscale variability of zero intermittency in spatial rainfall. J. Appl. Meteor., 33, 1516-1525.

Olssen, J. and R. Berndtsson, 1998: Temporal rainfall disaggregation based on scaling properties. Water Sci. Technol., 37 (11), $73-79$.

Onibon, H. and T. Lebel, 2004: Gibbs sampling for conditional spatial disaggregation of rain fields. Water Resour. Res., 40 (W08401), doi:10.1029/2003WR002009.

Ormsbee, L. E., 1986: Rainfall disaggregation model for continuous hydrological modeling. J. Hydraul. Eng., 115 (4), $507-525$.

Perica, S. and E. Foufoula-Georgiou, 1996: Model for multiscale disaggregation of spatial rainfall based on coupling meteorological and scaling descriptions. J. Geophys. Res., 101 (D21), 26347-26362.

Rebora, N., L. Ferraris, J. von Hardenberg, and A. Provenzale, 2006: RainFARM: Rainfall downscaling by a filtered autoregressive model. J. Hydrometeor., 7, 724-738.

Schleiss, M., J. Jaffrain, and A. Berne, 2011: Statistical analysis of rainfall intermittency at small spatial and temporal scales. Geophys. Res. Lett., 38, L18403, doi:doi:10.1029/2011GL049000.

Schleiss, M., J. Jaffrain, and A. Berne, 2012: Stochastic simulation of intermittent DSD fields in time. J. Hydrometeor., 13 (2), 621-637, doi:10.1175/JHM-D-11-018.1.

Sivakumar, B., S. Sorooshian, H. V. Gupta, and X. Gao, 2001: A chaotic approach to rainfall disaggregation. Water Resour. Res., 37 (1), 61-72.

Ulbrich, C. W., 1983: Natural variations in the analytical form of the raindrop-size distribution. J. Climate Appl. Meteor., 22 (10), 1764-1775.

Willis, P. T., 1984: Functional fits to some observed drop size distributions and parameterization of rain. J. Atmos. Sci., 41 (9), 1648-1661. 